\title{
Imaging the Activity of Immobilized Horse Radish Peroxidase with Scanning Electrochemical/chemiluminescence Microscopy
}

\author{
Huafang ZHOU, ${ }^{a}$ Shigenobu KASAI, ${ }^{\mathrm{b}}$ Tomoyuki YASUKAWA, ${ }^{\mathrm{b}}$ and Tomokazu MATSUE, ${ }^{\mathrm{a} *}$ \\ ${ }^{a}$ Center for Interdisciplinary Research and ${ }^{b}$ Graduate School of Engineering, Tohoku University (Sendai 980 - \\ 8578, Japan)
}

Received June 17, 1999 ; Accepted July 22, 1999

\begin{abstract}
A novel scanning electrochemical/chemiluminescence microscopy (SECM/SCLM) was used for imaging of a spot of immobilized horse radish peroxidase (HRP) on a substrate. When the SECM tip scanned over the spot in an aqueous luminol solution, the immobilized HRP catalyzed the oxidation of luminol by the electrogenerated $\mathrm{H}_{2} \mathrm{O}_{2}$ at the tip to emit chemiluminescence which was detected by a photon-counter. The photon-counting intensity was plotted against the position of the tip to give the image indicating the activity of immobilized HRP. The topography image was also obtained by mapping the oxygen reduction current, since the current was sensitive to the topography of substrate.
\end{abstract}

Key Words : Scanning Electrochemical/chemiluminescence Microscopy, Image, Horse Radish Peroxidase, Luminol

\section{Introduction}

Development of new methods and techniques to obtain information on spatial and temporal distributions of bio-molecules with a high sensitivities at microscale have evoked strong interests. ${ }^{1-5)}$ Owing to the simplicity and high sensitivity, detection of chemiluminescence by photon counter is one of the most often-used analytical methods for trace analysis of bio-samples. ${ }^{6-10)}$ However, photon counting detection does not provide detailed information on the localized distribution of chemiluminescence. Although image intensified CCD cameras $^{11,12)}$ can be used for imaging of localized chemiluminescence from solid substrate in a solution, it does not distinguish the target molecules from others on the substrate. We develop here a novel scanning electrochemical/ chemiluminescence microscopy system for imaging of localized redox current and chemiluminescence from microspots of immobilized horse radish peroxidase (HRP) at a glass substrate. The present system induces a chemiluminescence-generating reaction only in a limited area to provide simultaneously chemiluminescence and current images which indicate the activity and distribution and topography of the immobilized enzyme.

\section{Experimental}

The basic principles of SECM/SCLM are illustrated in Fig. 1. A carbon ultramicroelectrode (tip diameter, about $20 \mu \mathrm{m}$; electrode diameter, $8 \mu \mathrm{m}$ ) was attached to a high-precision motor-driven $\mathrm{XYZ}$ stage (Chuo Seiki, M 9103) placed on an inverted microscope (Nikon, TMD 200). The measurement of current image was carried out in a two-electrode configuration with a $\mathrm{Ag} / \mathrm{AgCl}$ (Saturated $\mathrm{KCl}$ ) counter electrode and a Keithley Model 428 amplifier. The XYZ stage control and the acquisition of current data were performed by a notebook computer through a GPIB interface (interface, AZI-3506). The acquisition of photon counter data was also done by the computer through a pulse counting board (Hamamatsu photonics M 3949). The photon counting and current images were obtained by mapping the photon counting and current data against the position of the ultramicroelectrode tip, respectively.

For imaging chemiluminescence and topography, horse radish peroxidase (HRP) was immobilized onto a glass substrate as follows. A clean glass substrate was dipped into a $10 \mathrm{mM}$ (3-aminopropyl)triethoxysilane/benzene solution for 8 hours followed by thorough rinse with water under surpersonication. A very small droplet (approximately $6 \mathrm{pL}$ ) of a $2.7 \times 10^{3}$ units/ $\mathrm{mL}$ HRP solution containing $1 \%(\mathrm{v} / \mathrm{v})$ glutaraldehyde and $5 \mathrm{mg} / \mathrm{mL}$ bovine serum albumin/water soluCarbon ultramicroelectrode

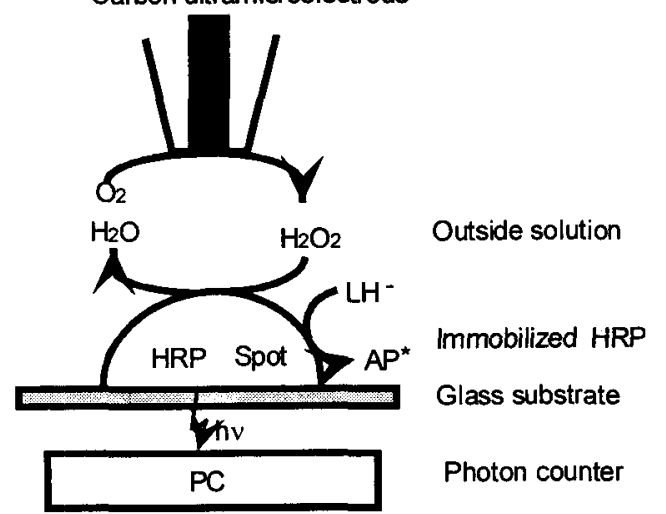

Fig. 1 Schematic diagram illustrating the operating principles of scanning electrochemical/chemiluminescence microscopy. 
tion was spotted on the aminosilanized substrate by glass capillary controlled with a motor driven actuator. The size of spot was approximately $30 \times 20$ $\mu \mathrm{m}$. After the immobilization of HRP, the substrate was dipped in a $2.0 \mathrm{mM}$ sodium luminol $(\mathrm{L} / \mathrm{HNa}) / 0.1$ $\mathrm{M} \mathrm{KCl}$ solution for the measurements. Then, the microelectrode probe $(-1.0 \mathrm{~V}$ vs. $\mathrm{Ag} / \mathrm{AgCl})$ scanned $(4.9 \mu \mathrm{m} / \mathrm{sec})$ two-dimensionally above the substrate in a constant height mode (tip-substrate distance, approximately $20 \mu \mathrm{m}$ ). At the tip dissolved oxygen was reduced to $\mathrm{H}_{2} \mathrm{O}_{2}$ which diffused onto the substrate. When the tip moves near the immobilized HRP, the HRP-catalyzed oxidation of LHNa by $\mathrm{H}_{2} \mathrm{O}_{2}$ generates chemiluminescence which is detected by the photon counter. The chemiluminescence image indicates the activity of the immobilized HRP. Since the reduction current of oxygen is influenced by the tipsubstrate distance, the current image indicates the topography of substrate.

\section{Results and Discussion}

Figure 2 shows an SCLM image of a substrate with a HRP spot $\left(1.8 \times 10^{-5}\right.$ units). The immobilized HRP was displayed as a bright spot with high chemiluminescence intensity. Because the intensity of the chemiluminescence is primarily influenced by the localized concentration and activity of HRP, the chemiluminescence image can provide the information on the enzyme activity. It should be noted that the size of the high intensity section (about $35 \times 30 \mu \mathrm{m}$ ) was slightly larger than the size of immobilized HRP spot (about $30 \times 20 \mu \mathrm{m}$ ), due to the diffusion of the electrocatalytically generated $\mathrm{H}_{2} \mathrm{O}_{2}$ over the spot and the dissolution of some HRP. Figure 3 shows an SECM image in the same scanning experiment of the case in Fig. 2. The brightest regions (lowest currents) indicate the immobilized HRP on the glass substrate. Since the immobilized HRP matrix swells into the
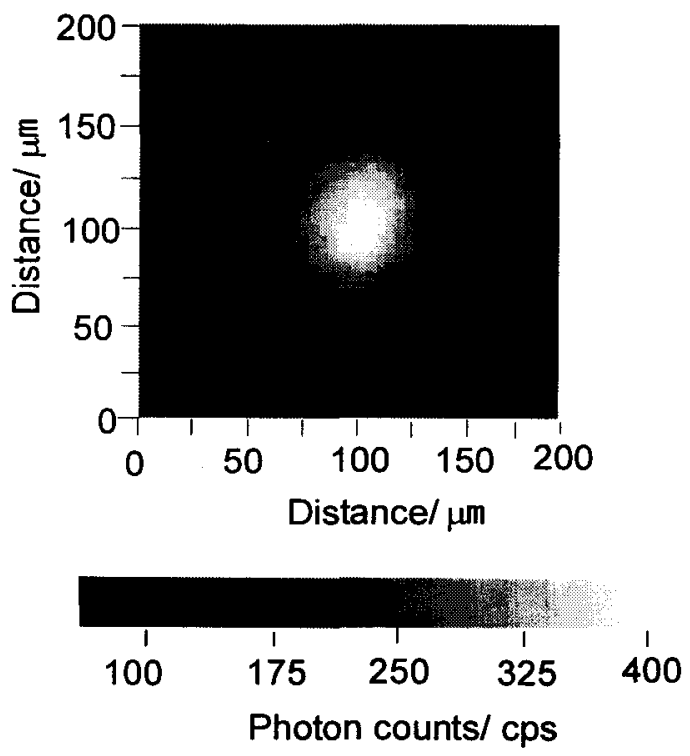

Fig. 2 SCLM image of HRP-immobilized substrate in 0.2 $\mathrm{M}$ phosphate buffer solution ( $\mathrm{pH} 8.7$ ) containing $2.0 \mathrm{mM}$ LHNa and $0.1 \mathrm{M} \mathrm{KCl}$. Scan rate of carbon ultramicroelectrode tip, $4.9 \mu \mathrm{m} / \mathrm{s}$. Tip height, $20 \mu \mathrm{m}$.

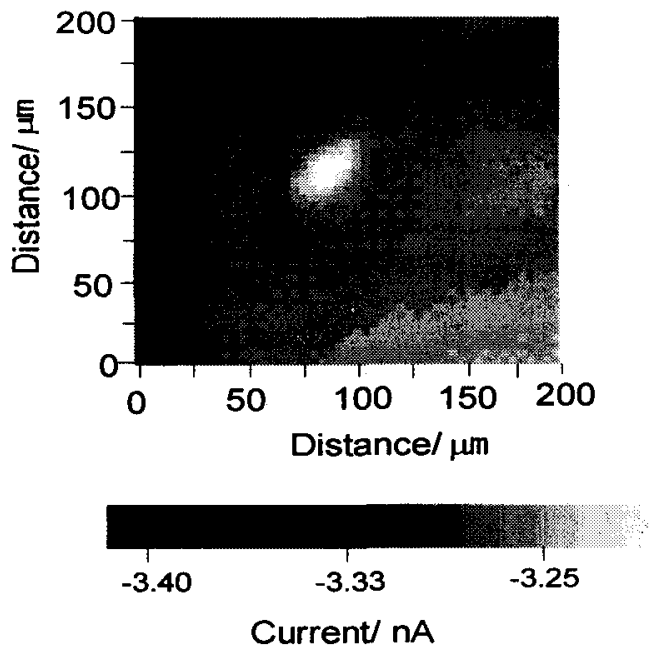

Fig. 3 SECM image of HRP-immobilized substrate in 0.2 $\mathrm{M}$ phosphate buffer solution $(\mathrm{pH} 8.7)$ containing $2.0 \mathrm{mM}$ LHNa and $0.1 \mathrm{M} \mathrm{KCl}$. Scan rate of the tip, $4.9 \mu \mathrm{m} / \mathrm{s}$. Tip height, $20 \mu \mathrm{m}$.

solution, the tip-substrate distance is short at the immobilized area compared to that at the bare area. The swelling matrix blocks the diffusion of oxygen from bulk solution to the tip surface and lowers the reduction current of oxygen. Thus, the image based on the oxygen reduction currents indicates the topography of the immobilized area.

In summary, we have developed a novel method of SECM/SCLM for characterization of immobilized enzymes. The chemiluminescence image indicates the activity and distribution of the immobilized enzyme, and the current image indicates the distribution of redox species and topography of the substrate. The two images of different basis are simultaneously obtained by using the present method. Since the scanning range is limited in microscale, undesired disturbing-reactions can be eliminated in the present system.

\section{Acknowledgment}

This work was partly supported by a Grant-in-Aid for Scientific Research on Priority Area (Electrochemistry of Ordered Interfaces, 09237106) from the Ministry of Education, Science and Culture, Japan.

\section{References}

1) S. Kasai, H. Zhou, and T. Matsue, submitted.

2) M. C. Messia, D. Compagnone, M. Esti, and G. Palleschi, Anal. Chem., 68, 360 (1996).

3) H. Meyer, H. Drewer, B. Grundig, K. Cammann, R. Kakerow, Y. Manoli, W. Mokwa, and M. Rospert, Anal. Chem., 67, 1164 (1995).

4) Y. Liu, T. Moroz, and J. V. Sweedler, Anal. Chem., 71, 28 (1999).

5) G. Nagy, C. X. Xu, R. P. Buck, E. Lindner, and M. R. Neuman, Anal. Chem., 70, 2156 (1998).

6) T. Hasebe, J. Naga, and T. Kawashima, Anal. Sci., 13, 93 (1997)

7) M. M. Collinson and R. M. Wightman, J. Phys. Chem., 
98, 11942 (1994).

8) R. Vogel and R. Sussmuth, Bioelectrochem. Bioenerg., 46, 65 (1998).

9) R. Vogel and R. Sussmuth, Bioelectrochem. Bioenerg., 48, 375 (1999).

10) P. M. Easton, A. C. Simmonds, A. Rakishev, A. M. Egorov, and L. P. Candeias, J. Am. Chem. Soc., 118,
6619 (1996).

11) A. Roda, P. Pasini, M. Musiani, S. Girotti, M. Baraldini, G. Arrea, and A. Suozzi, Anal. Chem., 68, 1073 (1996).

12) A. Dzgoev, M. Mecklenburg, P. O. Larsson, and B. Danielesson, Anal. Chem, 68, 3364 (1996). 